DOI: $10.17957 / \mathrm{IJAB} / 15.1700$

http://www.fspublishers.org

\title{
Comparative and Phylogenetic Analysis of Complete Chloroplast Genome Sequences of Two Wildrye Grasses Elymus sibiricus and $E$. nutans (Triticeae, Poaceae)
}

\author{
Qingqing Yu ${ }^{1 \dagger}$, Zhechuan Liu $^{1 \dagger}$, Yi Xiong ${ }^{1}$, Yanli Xiong ${ }^{1}$, Cong Nie ${ }^{1}$, Haidong Gao ${ }^{3}$, Wenhui Liu ${ }^{2}$ and Xiao Ma ${ }^{1 *}$ \\ ${ }^{1}$ College of Animal Science and Technology, Sichuan Agricultural University, Chengdu 611130, P. R. China \\ ${ }^{2}$ Qinghai Academy of Animal Science and Veterinary Medicine, Key Laboratory of Superior Forage Germplasm in the \\ Qinghai-Tibetan Plateau, Xi-ning, P. R. China \\ ${ }^{3}$ Genepioneer Biotechnologies Co. Ltd, Nanjing, 210023, P. R. China \\ *For correspondence: maroar@126.com \\ †Contributed equally to this work and are co-first authors \\ Received 30 December 2020; Accepted 01 March 2021; Published 10 May 2021
}

\begin{abstract}
Due to outstanding characteristics such as stress resistance and high biomass production, Elymus sibiricus (StH genomes) and E. nutans (StHY genomes) are regarded as ecologically important perennial bunchgrass species belonging to Elymus genus of tribe Triticeae (Poaceae), which were widely used to promote the restoration of degraded grassland in the eastern Tibetan Plateau. In this study, the complete chloroplast (cp) genome of E. sibiricus and E. nutans were sequenced and annotated with de novo analysis, to clarify their inter-species variation and their evolutionary relationships with relative species. The result showed that both two whole cp genomes shared a typical quadripartite structure, the cp genome length of $E$. sibiricus and $E$. nutans were $135,075 \mathrm{bp}$ and $135,060 \mathrm{bp}$, respectively. Three genes $t R N A-C G A, t R N A-C G U$, and $t R N A-C G U$ were unique in $E$. sibiricus while the gene $y c f l$ (hypothetical chloroplast reading frame no. 1) was only found in $E$. nutans. The identification of hotspot regions ( $t R N A-G U C \sim p s b M, t R N A-U A A \sim n d h J, r b c L \sim p s a I, \quad r p l 33 \sim r p s 18$ ) between the two cp genomes would be pertinent to the development of barcode marker of these two Elymus species. Comparative cp genome analysis and phylogenetic relationships further confirmed that Pseudoroegneria were putative matrilineal donors of St genome of Elymus species at plastome level. Whole cp genomes could be used as an effective barcode for species identification or for developing specific markers, which is essential useful for the evolutionary history and conservation of Elymus species. (C) 2021 Friends Science Publishers
\end{abstract}

Keywords: Elymus spp.; Chloroplast genome; Hotspot regions; Phylogenetic analysis

\section{Introduction}

Elymus L. is the largest genus of approximate 150 species of perennial grasses in the Triticeae tribe (Poaceae), also called wildrye and their species are widely distributed in most of the temperate regions in the world (Zhang et al. 2019). Given the high biomass, good forage quality and excellent tolerance to multiple biotic and abiotic stresses, the Elymus species are of great importance to the artificial grassland construction and degraded grassland restoration in northwestern China (Qiao et al. 2006). Furthermore, the excellent stress-resistance genes derived from Elymus species could be transferred to the related cereal crops for genetic improvement. E. sibiricus, with the genome constitution of StStHH $(2 \mathrm{n}=4 \mathrm{x}=28)$, along with $E$. nutans (StStHHYY, $2 n=6 x=42$ ), are the two most common perennial grasses species and widely used in forage production and restoring degraded grassland in the eastern Qinghai-Tibet Plateau (Zhang et al. 2016b). E. sibiricus usually has a lower drought resistance and higher biomass yield than E. nutans. However, the high morphological similarity and niche overlaps limit their germplasm identification and further hinders seed production and promotion (Lei et al. 2014). The sequencing of plastid genome via the next-generation sequencing technology (NGS) could provide a convenient and cost-effective approach to develop molecular markers, which was a potential tool for germplasms/species identification, evolutionary study, genetic relationship evaluation, and haplotype division derived from plastome (Li et al. 2014).

Chloroplast (cp) is an important component of plant organelles and photosynthetic organs (Hong et al. 2020). The cp genome was reported to be consisted of a typical quadripartite structure with a large single-copy region (LSC),

To cite this paper: Yu Q, Z Liu, Y Xiong, Y Xiong, C Nie, H Gao, W Liu, X Ma (2021). Comparative and phylogenetic analysis of complete chloroplast genome sequences of two wildrye grasses Elymus sibiricus and E. nutans (Triticeae, Poaceae). Intl J Agric Biol 25:1203-1212 
a small single-copy region (SSC) and two inverted repeat (IR) regions (Xiong et al. 2020; Hong et al. 2020). The cp genome of angiosperms is always $115-165 \mathrm{~kb}$ in length and contains about 130 genes, which are involved in photosynthesis, proteins encoding and transcriptional regulation (Daniell et al. 2016). Cp genome is not only necessary for the plant photosystem to promote photosynthesis and biomass yield, but also important in phylogenetic analysis and genetic diversity investigation due to its maternally inherited character and highly conserved genome sequences (Burke et al. 2012; Daniell et al. 2016; Zhang et al. 2016a). In particular, cp genome of plants is extraordinarily inherited from matrilineal line without interference of gene recombination, so its evolutionary path is correspondingly independent compared to the nuclear DNA (Ravi et al. 2008; Liu et al. 2018). The whole cp genome sequences and comparative analysis of some Triticeae species including genus Triticum, Aegilops, Pseudoroegneria, Hordeum, etc. (Gornicki et al. 2014; Chen et al. 2020). In addition, a point of concern is that Pseudoroegneria species with St-genome are generally considered the most likely maternal donor to Elymus L. genus, including E. sibiricus and E. nutans (Zuo et al. 2015). In this case, the comparative cp genome analysis between matrilineal lines and progeny species of Elymus would reveal their true evolutionary relationships.

The in-depth analysis of cp genome sequence for $E$. sibiricus and E. mutans is necessary to better understand the $\mathrm{cp}$ variance genes, structural variation of $\mathrm{cp}$ genomes and evolutionary relationships among Elymus species. Here, we present the de novo assembly and annotation of the $\mathrm{cp}$ genome sequence of E. sibiricus and E. mutans, and conduct a comparative analysis in order to (i) reveal the $\mathrm{cp}$ genomes variations between the two Elymus species and (ii) clarify the phylogenetic relationships between maternal species and progenies.

\section{Materials and Methods}

\section{Plant Material, DNA extracting, and chloroplast genome sequencing}

Fresh leaves of E. sibiricus (cv. Chuancao No.1) and $E$. nutans (cv. Aba) were collected in the country of Hongyuan, Aba Prefecture, Sichuan Province of China, located in southeastern Tibetan Plateau. Total DNA of one individual plant of each Elymus species were extracted using the Plant DNA Isolation Kit (ThermoFisher, Shanghai, China). Library construction and library quality testing were carried out after DNA quality was verified by $0.8 \%$ agarose gel. The chloroplast genomes were sequenced using Illumina Novaseq PE150 platform. The SPAdes v. 3.10.1 (Safonova et al. 2015) and Gapfiller v. 2.1.1 (Boetzer and Pirovano 2012) software were used to assemble the two studied Elymus cp genomes based on the reference cp sequence of Hordeum vulgare subsp. vulgare ( KT 962228.1) retrieved from NCBI database. In addition, the cp genome coding sequences (CDS) were compared against by Blast v. 2.2.25 (Kent 2002) pipeline and used for gene annotation. The rRNA and tRNA gene sequences of chloroplast genomes were compared and predicted by Hmmer v. $3.1 \mathrm{~b} 2$ software (Finn et al. 2011) and Aragorn v. 1.2.38 software (Laslett and Canback 2004), respectively. Lastly, Organellar Genome DRAW 1.3.1 (Lohse et al. 2013) was used to draw the circular cp genome map of Elymus.

\section{Alignments analysis of multiple chloroplast genomes}

The LAGAN mode of mVISTA (Poliakov et al. 2014) program was used to do multiple alignments of cp genomes between the two studied Elymus species, three Pseudoroegneria species, and Hordeum vulgare subspp. Vulgare, with E.sibiricus as reference. Homology and rearrangement occurrence of these species were analyzed in Mauve (Darling et al. 2010). Furthermore, the IRscope (Amiryousefi et al. 2018) online software was used to compare the boundary in the concatenation of IR and SC regions of the two Elymus, two Pseudoroegneria and Hordeum vulgare subsp. vulgare $\mathrm{cp}$ genomes.

\section{Recognition of repetitive sequences}

MISA v. 1.0 (Beier et al. 2017) software was used for extraction and recognition of the chloroplast Simple Sequence Repeats (cpSSRs). In addition, the inverted repetition (palindromic), direct repetition (forward), complement and reverse repetition with a minimum repetition length of $15 \mathrm{bp}$ and sequence consistency greater than $90 \%$ were searched by REPuter 3.0 software (Kurtz et al. 2001).

\section{Analysis of relative synonymous codon usage (RSCU)}

The MEGA v. 7.0 software was used to analyze the RSCU, which reflect the relative preference of specific bases encoding the corresponding amino acid codons (Kumar et al. 2016). Values of RSCU greater than one was considered as better codon usage frequency.

\section{Phylogenetic analysis and divergence time estimates}

Cp genome sequences of studied Elymus and fourteen Triticeae published species in the NCBI database were conducted for phylogenetic analysis using BEAST v. 1.7.3 package; with three Poaceae cp genomes as outgroup. The GenBank numbers of the relative species are listed in Table S1.

\section{Results}

Chloroplast genomes features of $E$. sibiricus and $E$. nutans

The cp genome of E. sibiricus and E. nutans were 


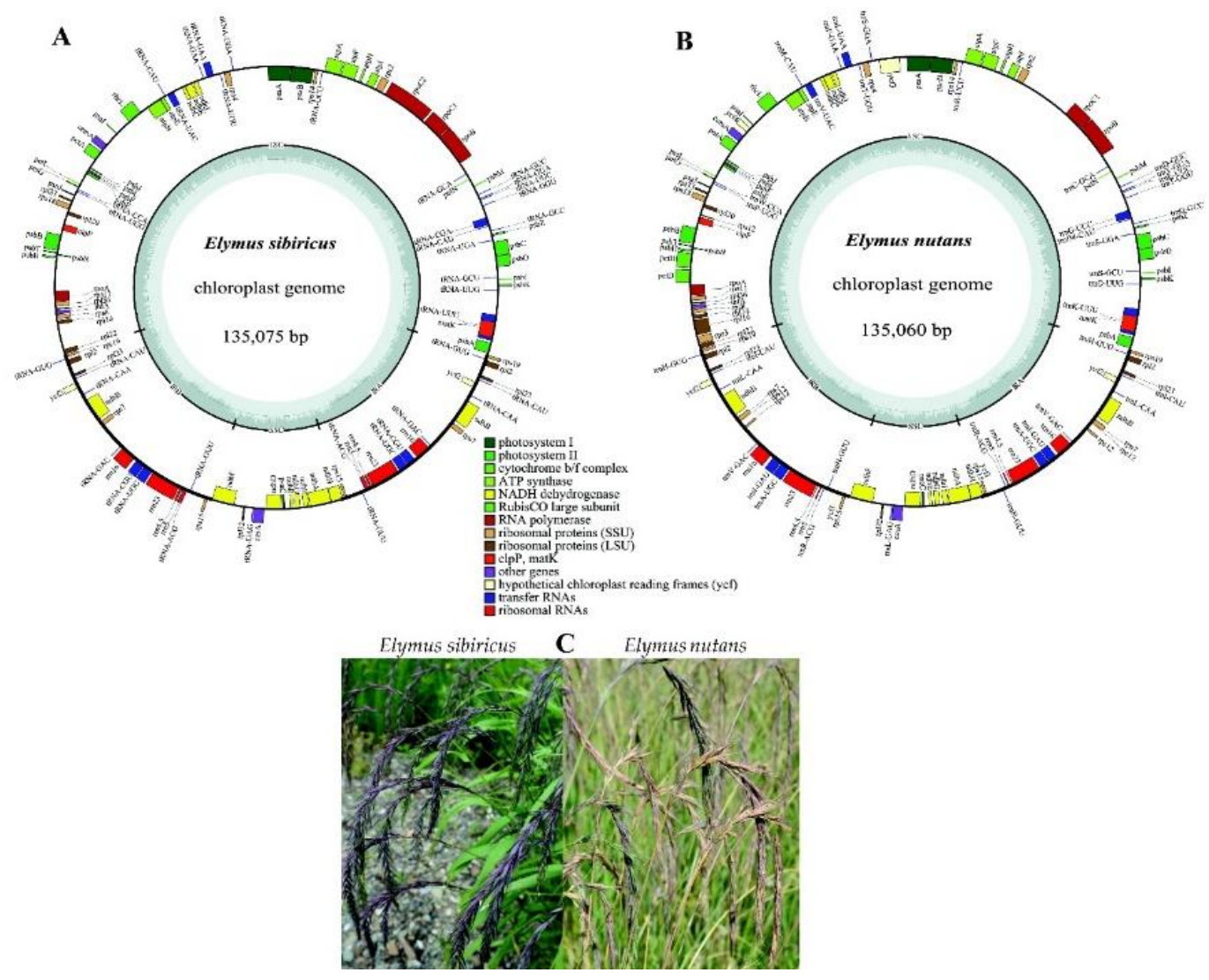

Fig. 1: Gene circle maps of the Elymus sibiricus (A) and Elymus nutans (B) chloroplast genomes. Genes belonging to different functional groups are color-coded. Genes transcribed clockwise and counterclockwise are indicated on the outside and inside of the large circle, respectively. The darker gray in the inner circle corresponds to GC content, whereas the lighter gray corresponds to AT content. Panicle morphology of two Elymus species (C)

sequenced and de novo assembled using Illumina short reads produced by genome skimming. The whole cp genome of E. sibiricus and E. nutans is $135,075 \mathrm{bp}$ and $135,060 \mathrm{bp}$, respectively. Both their genomes have a typical quadripartite structure. Their cp DNA were divided into a LSC region of 80,681 bp and 80,658 bp, an SSC region of $12,768 \mathrm{bp}$ and $12,766 \mathrm{bp}$ and two IR regions of $20,813 \mathrm{bp}$ and 20,818 bp (Fig. 1 and Table 1). The guanine and cytosine (GC) contents of the cp genomes appeared very similar between E. sibiricus $(38.34 \%)$ and $E$. nutan (38.33\%).

The complete cp genomes of E. sibiricus and $E$. nutans contained 102 and 109 genes, respectively. Both cp genomes had four rRNAs, except that number of both tRNA and mRNA in E. sibiricus cp genome was slightly lower than E. nutans (Table 1). A total of 20 and 21 duplicated genes were found in IR of E. sibiricus and $E$. nutans, respectively. Two tRNA genes ( $t R N A-C G A$ and $t R N A-C G U$ ) only existed in E. sibiricus and three tRNA genes (trnI-CAU, trnG-UCC and trnI-GAU) were only found in E. nutans (Table 2 and 3). In the 47 photosynthesis-related genes, four genes $(y c f 3, y c f 4$, petB and petD) were unique to $E$. nutans. Among genes associated with encoding ribosomal proteins and transcription, rps3, rps 12, and rpl16 were specific in $E$. nutans while rpl32 and rpoC2 were unique to E. sibiricus. Additionally, only one pseudogenized $y c f l$ gene was found in E. nutans (Table 3).

\section{SSRs (simple sequence repeats) and interspersed repetitive sequences analysis}

Interspersed repetitive (IR) sequences include palindrome repeats (P) and direct repeats (D). A total of 228 IR sequences were detected in the cp genome of $E$. nutans, which was higher than that of E. sibiricus (211). The percentage of type P repeats $(48.25 \%$, Fig. 2$)$ in E. nutans was slightly lower than E. sibiricus (49.25\%), but the type D repeats in $E$. nutans $(51.75 \%)$ was slightly higher than $E$. sibiricus $(50.7 \%)$.

A total of 165 and 161 SSRs were detected in cp genome of $E$. sibiricus and $E$. nutans, respectively. The single-bases $\mathrm{A}$ and $\mathrm{T}$ have the greatest number of repeat motifs in the two Elymus species (Fig. S1). A percentage of 77.0, 9.7 and $13.3 \%$ of SSRs were detected in LSC, SSC, and IR region of E. sibiricus (Fig. 3B). A very similar percentage pattern was found in E. nutans (Fig. 3C). In total, 
Yu et al. / Intl J Agric Biol, Vol 25, No 6, 2021

Table 1: Comparison of the sequenced cp genomes of the two Elymus species

\begin{tabular}{|c|c|c|}
\hline \multirow[t]{2}{*}{ Sequence region } & \multicolumn{2}{|c|}{ Length (bp) } \\
\hline & E. sibiricus & E. nutans \\
\hline Total cp genome & 135075 & 135060 \\
\hline LSC region & 80681 & 80658 \\
\hline SSC region & 12768 & 12766 \\
\hline IR region & 20813 & 20818 \\
\hline GC content & Percentage (\%) & \\
\hline Total cp genome & 38.34 & 38.33 \\
\hline LSC region & 36.37 & 36.37 \\
\hline SSC region & 32.32 & 32.24 \\
\hline IR region & 44 & 43.99 \\
\hline Gene Classification & Number & \\
\hline Total genes & 28 & 29 \\
\hline tRNA genes & 4 & 4 \\
\hline rRNA genes & 70 & 76 \\
\hline mRNA genes & 102 & 109 \\
\hline Number of genes duplicated in IR & 20 & 21 \\
\hline
\end{tabular}

Table 2: Location and length of genes containing intron in two chloroplast genomes

\begin{tabular}{|c|c|c|c|c|c|c|c|c|}
\hline \multirow[t]{2}{*}{ Gene } & \multicolumn{4}{|c|}{ E. sibiricus } & \multicolumn{4}{|c|}{ E. nutans } \\
\hline & Location & Exon I (bp) & Intron I (bp) & Exon II (bp) & Location & Exon I (bp) & Intron I (bp) & Exon II (bp) \\
\hline atpF & LSC & 158 & 803 & 409 & LSC & 144 & 819 & 407 \\
\hline$n d h A$ & SSC & 550 & 1026 & 539 & SSC & 550 & 1026 & 539 \\
\hline$n d h B$ & IRB & 777 & 712 & 756 & IRB & 777 & 712 & 756 \\
\hline$t R N A-C G A$ & LSC & 32 & 662 & 63 & LSC & - & - & - \\
\hline$t R N A-C G U$ & IRA & 32 & 787 & 59 & IRA & - & - & - \\
\hline$t R N A-C G U$ & IRB & 33 & 785 & 60 & IRB & - & - & - \\
\hline$t R N A-U A A$ & LSC & 36 & 575 & 51 & LSC & 35 & 574 & 50 \\
\hline$t R N A-U A C$ & LSC & 39 & 579 & 54 & LSC & 39 & 596 & 37 \\
\hline$t R N A-U G C$ & IRA & 37 & 811 & 36 & IRA & 37 & 811 & 35 \\
\hline$t R N A-U G C$ & IRB & 38 & 809 & 37 & IRB & 37 & 811 & 35 \\
\hline$t R N A-U U U$ & LSC & 39 & 2485 & 37 & LSC & 37 & 2488 & 35 \\
\hline
\end{tabular}

Table 3: Comparison of the two Elymus species' chloroplast (cp) genomes

\begin{tabular}{|c|c|c|}
\hline Category & Function & Name of genes \\
\hline \multirow[t]{2}{*}{ Self-replication (35) } & Ribosomal RNA Genes & $r r n 4.5, r r n 5, r r n 16, r r n 23$ \\
\hline & Transfer RNA genes & $\begin{array}{l}\operatorname{trn} A-A C G, \operatorname{trn} A-C A A, \operatorname{trn} A-C A U, \operatorname{trn} A-C C A, \operatorname{trn} A-G A A, \operatorname{trn} A-G A C, \operatorname{trn} A-U A G, \operatorname{trn} A-U A C^{*}, \\
\operatorname{trn} A-U U G, \operatorname{trn} A-U U U^{*}, \operatorname{trn} A-G C A, \operatorname{trn} A-G C C, \operatorname{trn} A-G G U, \operatorname{trn} A-G U A, \operatorname{trn} A-G U C, \operatorname{trn} A-G U G, \\
\operatorname{trn} A-U G A, \operatorname{trn} A-U G C^{*}, \operatorname{trn} A-U G U, \operatorname{trn} A-U G G, \operatorname{trn} A-C G A^{* / e s}, \operatorname{trn} A-U C U, \operatorname{trn} A-G C U, \operatorname{trn} A-G U U, \\
\operatorname{trn} A-C G U^{* / e s}, \operatorname{trn} A-U U C, \operatorname{trn} A-G G A, \operatorname{trn} A-U A A^{*}, \operatorname{trn} I-C A U^{\text {en }}, \operatorname{trn} G-U C C^{* / e n}, \operatorname{trn} I-G A U^{* / e n}\end{array}$ \\
\hline $\begin{array}{l}\text { Ribosomal proteins (11) } \\
\text { (translation) }\end{array}$ & Small subunit of ribosome (SSU) & $r p s 2, r p s 3^{\text {en }}, r p s 4, r p s 7, r p s 8, r p s 11, r p s 12^{* / e n}, r p s 14, r p s 15, r p s 16, r p s 18, r p s 19$ \\
\hline \multirow[t]{2}{*}{ Transcription (14) } & Large subunit of ribosome (LSU) & rpl2, rpl14, rpl16 $6^{* / e n}, r p l 20, r p l 22, r p l 23, r p l 32^{\mathrm{es}}, \mathrm{rpl33}, \mathrm{rpl36}$ \\
\hline & Translation initiation factor & infA \\
\hline \multirow{7}{*}{$\begin{array}{l}\text { Photosynthesis } \\
\text { genes (47) }\end{array}$} & Large subunit of Rubisco & $r b c L$ \\
\hline & Subunits of Photosystem I & psaA, psaB, psaC, psaI, psaJ, ycf3 $3^{* * / e n}, y c f 4^{\mathrm{en}}$ \\
\hline & Subunits of Photosystem II & $p s b A, p s b B, p s b C, p s b D, p s b E, p s b F, p s b H, p s b I, p s b J, p s b K, p s b L, p s b M, p s b T, p s b Z, p s b N$ \\
\hline & Subunits of ATP synthase & atpA, atpB, atpE, atpF $F^{*}$ atpH, atpI \\
\hline & Cytochrome b/f complex & petA, pet $B^{* / e n}$, pet ${ }^{* / e n}, \operatorname{pet} G, \operatorname{pet} L, \operatorname{pet} N$ \\
\hline & C-type cytochrome synthesis gene & $\operatorname{ccs} A$ \\
\hline & Subunits of NADH dehydrogenase & $n d h A^{*}, n d h B^{*}, n d h C, n d h D, n d h E, n d h F, n d h G, n d h H, n d h I, n d h J, n d h K$ \\
\hline \multirow{3}{*}{ Other genes (5) } & Chloroplast envelope membrane protein & cemA \\
\hline & Hypothetical protein & $y c f 1^{\mathrm{en}}$ \\
\hline & Hypothetical open reading frames & $y c f 2$ \\
\hline
\end{tabular}

Note: Asterisk denotes the genes including a single intron; two asterisks denote the genes including two introns; ${ }^{\text {es }}$, genes that are unique for E. sibiricus; ${ }^{\text {en }}$, genes that are unique for $E$. nutans

71 SSRs existed in the exon region of E. sibiricus, while only 52 were found in the exon region of $E$. nutans. At the SSC region, there were six intergenic SSRs found only in $E$. nutans (Fig. 3A).

\section{Feature of IR scope}

The contraction and expansion of IR region were compared in the $\mathrm{cp}$ genomes of $E$. sibiricus, $E$. nutans, 


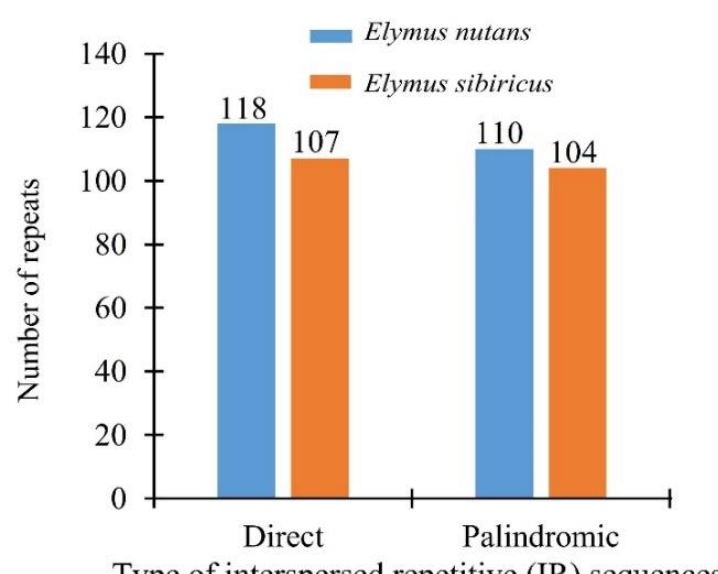

Type of interspersed repetitive (IR) sequences

Fig. 2: Type and number distribution of repeat sequences in $\mathrm{cp}$ genomes of two Elymus species

Pseudoroegneyia spicata, P. libanotica and Hordeum vulgare subsp. vulgare (Fig. 4). Overall, the result suggested little difference in the junction positions among the Elymus and Pseudoroegneyia cp genome sequences. There is a 34 bp spacer between $r p l 22$ genes and JBL (junction position of LSC and IRb region) in E. sibiricus, $P$. spicata and $P$. libanotica, whereas only 29 bp spacer was detected in $E$. nutans. Similarly, rps 19 gene and JLA (junction position of LSC and IRa region) were separated by a 48 bp spacer in $E$. sibiricus, $P$. spicata and $P$. libanotica and 53 bp spacer in $E$. nutans. More specifically, the gene $y c f l$ was only detected in the IRa region of $E$. nutans and $P$. libanotica.

\section{Variation analysis of six chloroplast genomes}

The genetic variation among the two Elymus species, Hordeum vulgare ssp. vulgare and three Pseudoroegneyia cp genomes were analyzed via mVISTA (Poliakov et al. 2014) and Mauve (Darling et al. 2010). The results of the mVISTA revealed a lower variance in SSC and IR regions than in LSC regions, and more conservation in the coding regions than the non-coding regions (Fig. 5). The variation hotspot mainly existed in intragenic region. At the whole cp genome level, only a few variation hotspot regions existed in Elymus and Pseudoroegneyia species, which included tRNA-GUC psbM, tRNA-UAA ndhJ, rbcL psaI, rpl33 rps 18, and so on (Fig. 5). The result of the mVISTA analysis only between $E$. sibiricus and $E$. nutans shown that there were several hotspot regions (tRNA-GUC psbM, tRNA-UAA ndhJ, rbcL psal, rpl33 rps18, and so on). However, as shown in the local collinear block (Fig. S2), no inversion events or rearrangement were found among the six related species.

\section{Analysis of relative synonymous codon usage}

Relative synonymous codon usage (RSCU) is considered a

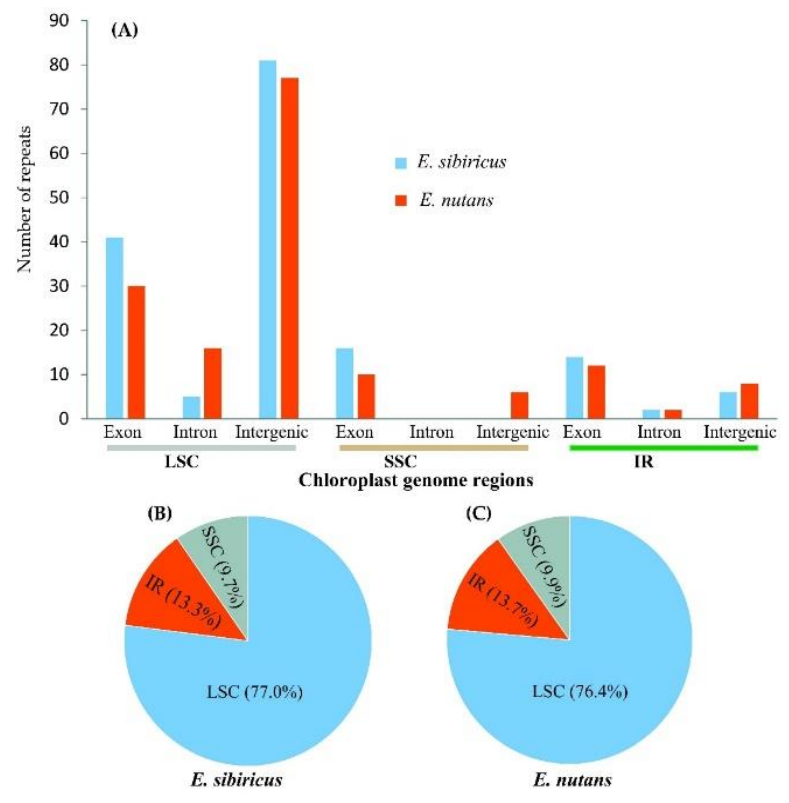

Fig. 3: Number (A) and frequency $(\mathbf{B}, \mathbf{C})$ of SSRs in the different region of Elymus cp genome

combination of natural selection, genetic drift, and mutation. The RSCU of the two Elymus cp genomes was analyzed based on the 66 shared protein-coding genes (Fig. S3). We found that the RSCU values of initiation codon AUG were 1.991 and 1.982 in E. sibiricus and E. nutans, respectively. For three termination codons UAA, UAG, and UGA, the RSCU values were 1.6941, 0.6354 and 0.6705 in $E$. sibiricus and 1.7922, 0.5844 and 0.6234 in E. nutans. When the RSCU value of the codon was greater than one, it was considered a larger codon frequency. A $48.48 \%$ percentage (32 of 66, including three termination codons) of codons showed a greater frequency than one (RSCU > 1) both of two Elymus species, where $90.63 \%$ (29 of 32) codons prefer $\mathrm{A}+\mathrm{U}$ at the third position.

\section{Phylogenetic tree and divergence time}

The Maximum-likelihood (ML) phylogenetic tree, based on the Bayesian MCMC (Markov Chain Monte Carlo) method, was obtained using the whole cp genome sequences of nineteen Poaceae species and, Saccharum spontaneum, Sorghum bicolor, and Avena sativa as outgroups (Fig. 6). Clearly, phylogenetic analysis supported the traditional phylogenetic classification of the Triticeae tribe. Two studied Elymus species and three Pseudoroegneria species were grouped in one clade, in which $E$. sibiricus, E. nutans, and three Pseudoroegneria species diverged around 3.061 Mya ago (Fig. 6). Approximately at 0.5746 Mya, E. sibiricus, $P$. libanoticus and $P$. tauri were divided, and around 0.4664 Mya the E. nutans and P. spicata were spitted from each other (Fig. 6), thus suggesting a close phylogenetic relationship between $E$. nutans and $P$. spicata. 
Yu et al. / Intl J Agric Biol, Vol 25, No 6, 2021

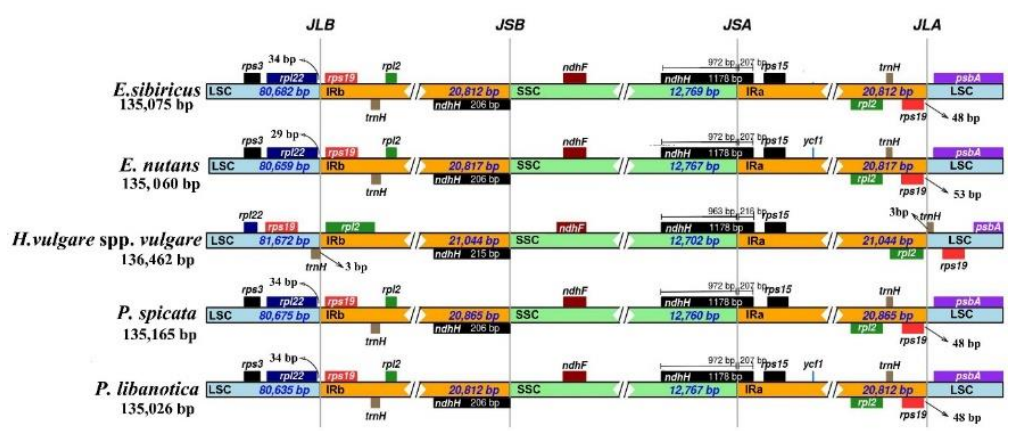

Fig. 4: IR scope analysis of cp genomes of five species. JLB, the junction position of LSC and IRb region; JSB, the junction position of SSC and IRb region; JSA, the junction position of SSC and IRa region; JLA, the junction position of LSC and IRa region
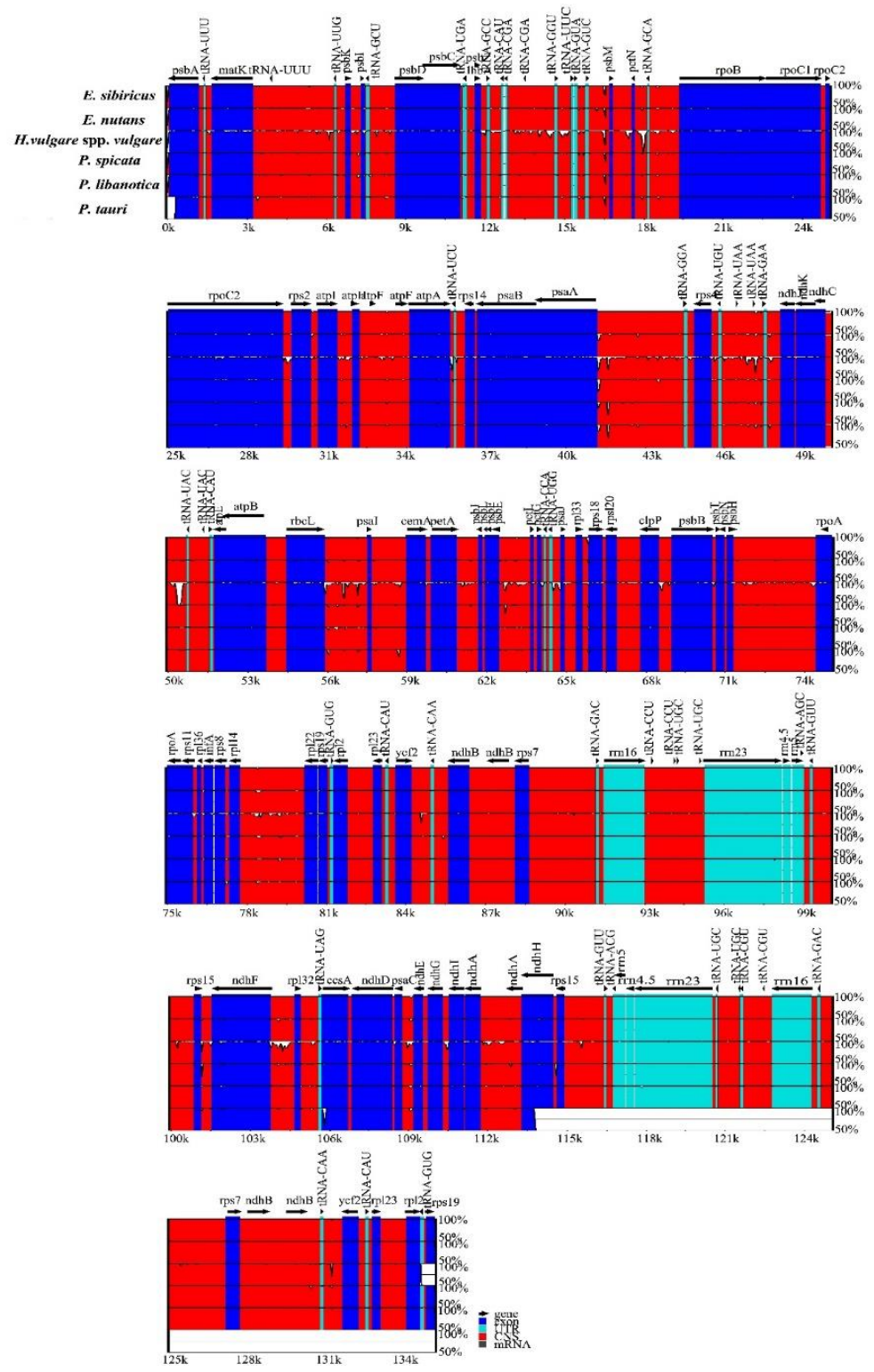

Fig. 5: Sequence identity plots among the two Elymus species and three Pseudoroegneyia species, with E. sibiricus as a reference. Annotated genes are shown on the top. Genome regions are color-marked as CNS (conserved non-coding sequences), exons, and introns. The color legend is summarized in the lower right-hand corner. Vertical scale indicates the percentage of identity ranging from $50 \%$ to $100 \%$ 


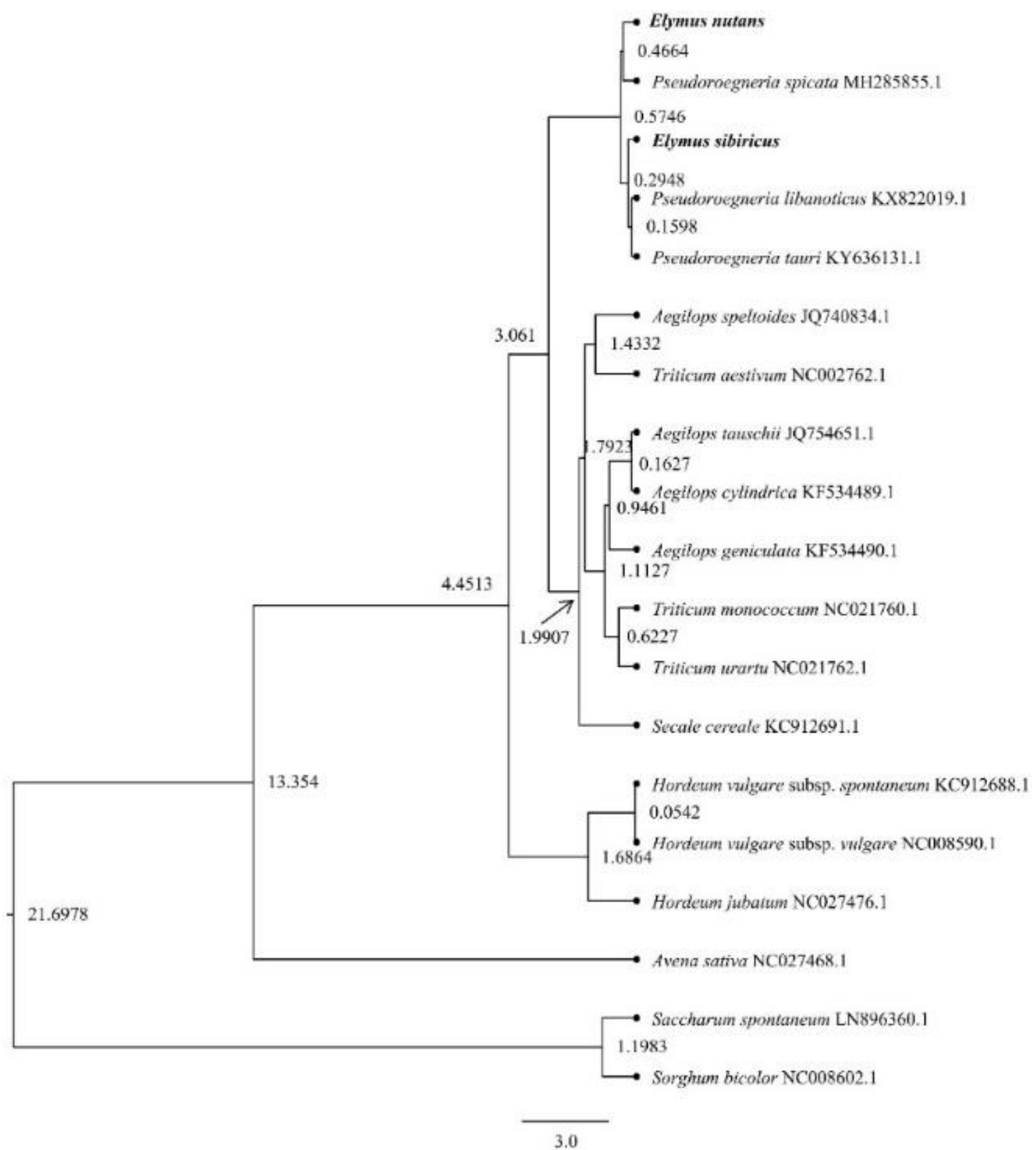

Fig. 6: Phylogenetic tree and divergence time among nineteen chloroplast genomes, the node value of the tree represents the average divergence time. The species used in this study are bolded

\section{Discussion}

Regularly, the 74 protein-coding genes were found in most angiosperms, while an additional five were found only in some species (Raman and Park 2016). However, 76 and 70 protein-coding genes were detected in E. nutans and $E$. sibiricus, respectively. These differential genes (e.g., ycfl, $y c f 3, y c f 4, r p s 3, r p s 12, r p l 32)$ between the two Elymus species might be completely lost or transferred to the nuclear genome (Kan et al. 2020). In details, a unique pseudogenized $y c f l$ gene was found only to exist in $E$. nutans and Pseudoroegneyia libanotica. The ycfl gene is functional and essential for cell survival in cp genomes of dicots except Poaceae (Huang et al. 2017). It is possible that the $y c f l$ gene is not necessary for evolution and similarly to the tufA gene in angiosperms (Turmel et al. 2007), functionality of $y c f l$ gene was transferred to the nuclear genome of those species that have one $y c f$ pseudogene. Moreover, the $t R N A-C G A$ in the LSC region and $t R N A$ $C G U, t R N A-C G U$ in the IR region of $E$. nutans cp genome 
have been lost. Although the cp genome of Poaceae is tremendously conservative, the subsistent differences will provide the basis for understanding the unique differences between related species or subspecies (Xiong et al. 2020).

Vast variant boundary regions of LSC/IRb, IRb/SSC, $\mathrm{SSC} / \mathrm{IRa}$, and IRa/LSC are responsible for variations in $\mathrm{cp}$ genome size and rearrangement (Li et al. 2017). In addition, the rpl22 gene, with the function of regulating senescence to maintain cell viability (Toro et al. 2019), showed a tendency of moving toward the IRb region in E. nutans compared with E. sibiricus and two Pseudoroegneyia species. It is well known that the most conservative quadripartite structure in the cp genome were the IR regions (Xiong et al. 2020). Therefore, this drift might help rpl22 gene transfer into the IR region and further maintain the stability to attain the evolutionary adaptation of $E$. nutans.

CpSSR is one of the most significant tools to study genetic diversity, variety identification and phylogenetic analysis (Yamane and Kawahara 2018). Particularly, cp genomes have ancient patterns of inheritance that can offer insights into the evolutionary process (Cremen et al. 2018). Thus, the difference of cpSSRs in two Elymus could be used to further identify intraspecific genetic polymorphism. Except for cpSSR, many different cp DNA fragments and hotspot mutations could be used to develop barcode markers for congeneric species. There were many scattered mutational events existing in the $\mathrm{cp}$ genomes, which were generally gathered in "hotspots" and leaded a high variation region to distinguish the related species (Chao et al. 2017). In the two Elymus species we identified several hotspots regions, among them $t R N A-G U C \sim p s b M$, tRNA-UAA ndhJ, rbcL psaI, rpl33 rps18, which could be used as new potential markers for future phylogenetic and phylogeographic studies of Elymus species if available. Among these highly variable regions, the region of rpl33 rps 18 has been used as DNA barcodes in some plant species (Mariotti et al. 2010).

The RSCU values were calculated using the common genes of two Elymus species. The codon of leucine revealed the highest frequency (RSCU > 2), whereas the lowest frequency was found in the codon of methionine (RSCU < 0.02 ). The result was consistent with previous studies on $\mathrm{cp}$ genome of angiosperms ( $\mathrm{Li}$ et al. 2019). Additionally, in agreement with Premal (Shah and Gilchrist 2011), we found that almost all of the codons with a high RSCU (RSCU > 1) value were $\mathrm{A} / \mathrm{U}$ ended.

Chloroplast genome plays an important role in the evolutionary study due to the conservation of maternal inheritance (Nielsen et al. 2013). In this study, to obtain a more accurate evolutionary relationship and divergence time between $E$. sibiricus and E. nutans, their whole cp genome and other fifteen related species were used. The result showed that the Elymus and Pseudoroegneyia species separated from other Triticeae species about 3.061 million years ago (Mya). However, it is interesting to note that the $E$. sibiricus and $E$. nutans were grouped in two separate branches (Fig. 6). According to Chen et al. 2020, the shared
St genome of E. sibiricus and E. nutans was both inherited from the Pseudoroegneyia species, while the respective specific species is not unambiguous. The phylogenetic relationships obtained in this study indicates that $E$. natans was more closely related to Pseudoroegneyia spicata, while $E$. sibiricus was closely related to $P$. libanoticus and $P$. tauri. Here, we could get a preliminary suggestion that the St nuclear genome of $E$. sibiricus originate from $P$. libanoticus or $P$. tauri and the St genome of $E$. natans originate from $P$. spicata. Of course, more evidence from nuclear genomes is required to support this view.

\section{Conclusion}

In present study, sequencing and de novo assembly of chloroplast genomes of E. sibiricus and E. nutans (Poaceae, Triticeae) were conducted using Illumina sequencing platform, which is an advantageous tool to research the origin and evolution of Elymus genus. We found that the structural characteristics of the two Elymus species have typical four-part structure in relationships similar to other Poaceae species. Large differences of interspersed repetitive sequences were detected between the two Elymus species. In addition, several hotspots (e.g., tRNAGUC psbM, tRNA-UAA ndhJ, rbcL psaI, rpl33 rps18) could be used to develop barcode marker for Elymus species. Finally, the phylogenetic analysis was in accordance with the traditional phylogenetic classification of the Triticeae tribe. This study provided new plastome insights into phylogenetic status and valuable gene resource in Elymus genus of Triticeae tribe.

\section{Acknowledgments}

We are very grateful to the Department of Grassland Science, Sichuan Agricultural University for providing us with experimental equipment and venue. Thanks to all authors for their hard work on this manuscript.

\section{Funding}

This work was supported by the Open project of the Key Laboratory of Utilization of Excellent Forage Germplasm Resources in Qinghai-Tibet Plateau of Qinghai Province (2020-ZJ-Y03) and Sichuan Province College Students' innovation and entrepreneurship training program (2019008006).

\section{Grant Disclosures}

The grant information was disclosed by the authors as "Open project of the Key Laboratory of Utilization of Excellent Forage Germplasm Resources in Qinghai-Tibet Plateau of Qinghai Province: 2020-ZJ-Y03. Sichuan Province College Students' innovation and entrepreneurship training program: 2019008006" 


\section{Author Contributions}

Conceptualization, Xiao Ma; Methodology, Qingqing Yu and Zhechuan Liu; Resources, Wenhui Liu and Haidong Gao; Software, Yi Xiong, Zhechuan Liu and Yanli Xiong; Writing - original draft, Qingqing Yu and Xiao Ma; Writing - review \& editing, Qingqing Yu, Yi Xiong, Cong Nie and Wenhui Liu.

\section{Conflict of Interest}

The authors declare there are no competing interests.

\section{Data Availability}

The annotated chloroplast genomes of Elymus sibiricus and E. nutans have been deposited in the NCBI GenBank with the accession numbers MT610375 and MT610376.

\section{Ethics Approval}

Not applicable.

\section{References}

Amiryousefi A, J Hyvonen, P Poczai (2018). IRscope: An online program to visualize the junction sites of chloroplast genomes. Bioinformatics 34:3030-3031

Beier S, T Thiel, T Münch, U Scholz, M Mascher (2017). MISA-web: A web server for microsatellite prediction. Bioinformatics 16:25832585

Boetzer M, W Pirovano (2012). Toward almost closed genomes with GapFiller. Genom Biol 13:1-9

Burke SV, CP Grennan, MR Duvall (2012). Plastome sequences of two New World bamboos-Arundinaria gigantea and Cryptochloa strictiflora (Poaceae)-extend phylogenomic understanding of Bambusoideae. Amer J Bot 99:1951-1961

Chao X, D Wenpan, L Wenqing, L Yizeng, X Xiaoman, J Xiaobai, S Jipu, H Kaihong, S Zhili (2017). Comparative analysis of six Lagerstroemia complete chloroplast genomes. Front Plant Sci 8; Article 15

Chen N, W Chen, H Yan, Y Wang, H Kang, H Zhang, Y Zhou, G Sun, L Sha, X Fan (2020). Evolutionary patterns of plastome uncover diploid-polyploid maternal relationships in Triticeae. Mol Phylogenet Evol 149:106838

Cremen MCM, F Leliaert, VR Marcelino, H Verbruggen (2018). Large diversity of nonstandard genes and dynamic evolution of chloroplast genomes in Siphonous Green Algae (Bryopsidales, Chlorophyta). Genom Biol Evol 10:1048-1061

Daniell H, C Lin, M Yu, W Chang (2016). Chloroplast genomes: Diversity, evolution, and applications in genetic engineering. Genome Biol $17: 134-162$

Darling AE, B Mau, NT Perna (2010). ProgressiveMauve: Multiple genome alignment with gene gain, loss and rearrangement. PLoS One 5; Article e11147

Finn RD, J Clements, SR Eddy (2011). HMMER web server: Interactive sequence similarity searching. Nucl Acids Res 39:29-37

Gornicki P, H Zhu, J Wang, GS Challa, Z Zhang, BS Gill, W Li (2014). The chloroplast view of the evolution of polyploid wheat. New Phytol 204:704-714

Hong Z, Z Wu, K Zhao, Z Yang, N Zhang, J Guo, LR Tembrock, D Xu (2020). Comparative analyses of five complete chloroplast genomes from the genus Pterocarpus (Fabacaeae). Intl J Mol Sci 21:3758-3775
Huang YY, ST Cho, M Haryono, CH Kuo (2017). Complete chloroplast genome sequence of common bermudagrass (Cynodon dactylon (L.) Pers.) and comparative analysis within the family Poaceae. PLoS One 12; Article $\mathrm{e} 0179055$

Kan S, T Shen, P Gong, J Ran, X Wang (2020). The complete mitochondrial genome of Taxus cuspidata (Taxaceae): Eight protein-coding genes have transferred to the nuclear genome. BMC Evol Biol 20; Article 10

Kent WJ (2002). BLAT-The BLAST-like alignment tool. Genomics Res 12:656-664

Kumar S, G Stecher, K Tamura (2016). MEGA7: Molecular evolutionary genetics analysis version 7.0 for bigger datasets. Mol Biol Evol 33:1870-1874

Kurtz S, JV Choudhuri, E Ohlebusch, C Schleiermacher, J Stoye, R Giegerich (2001). REPuter: The manifold applications of repeat analysis on a genomic scale. Nucl Acids Res 29:4633-4642

Laslett D, B Canback (2004). ARAGORN, a program to detect $t R N A$ genes and tmRNA genes in nucleotide sequences. Nucl Acids Res 32:11-16

Lei Y, Y Zhao, F Yu, Y Li, Q Dou (2014). Development and characterization of 53 polymorphic genomic-SSR markers in Siberian wildrye (Elymus sibiricus L.). Conserv Genet Resour 6:861-864

Li Y, SP Sylvester, M Li, C Zhang, X Li, Y Duan, X Wang (2019). The complete plastid genome of Magnolia zenii and genetic comparison to Magnoliaceae species. Molecules 24:261-276

Li Y, Z Hansheng, P Zhenhua, L Dong, Z Gao (2014). Development and application of SSR molecular markers from the chloroplast genome of bamboo. J Trop Subtrop Bot 3:263-269

Li Z, H Long, L Zhang, Z Liu, H Cao, M Shi, X Tan (2017). The complete chloroplast genome sequence of tung tree (Vernicia fordii): Organization and phylogenetic relationships with other angiosperms. Sci Rep 7; Article 1869

Liu L, Y Wang, P He, P Li, J Lee, DE Soltis, C Fu (2018). Chloroplast genome analyses and genomic resource development for epilithic sister genera Oresitrophe and Mukdenia (Saxifragaceae), using genome skimming data. BMC Genomics 19; Article 235

Lohse M, O Drechsel, S Kahlau, R Bock (2013). Organellar Genome DRAW - a suite of tools for generating physical maps of plastid and mitochondrial genomes and visualizing expression data sets. $\mathrm{Nucl}$ Acids Res 41:575-581

Mariotti R, NGM Cultrera, CM Diez, L Baldoni, A Rubini (2010). Identification of new polymorphic regions and differentiation of cultivated olives (Olea europaea L.) through plastome sequence comparison. BMC Plant Biol 10; Article 211

Nielsen AZ, B Ziersen, K Jensen, LM Lassen, CE Olsen, BL Moller, PE Jensen (2013). Redirecting photosynthetic reducing power toward bioactive natural product synthesis. ACS Synth Biol 2:308-315

Poliakov A, J Foong, M Brudno, I Dubchak (2014). GenomeVISTA - an integrated software package for whole-genome alignment and visualization. Bioinformatics 30:2654-2655

Qiao AH, JG Han, AQ Gong, W Li, YW Wang, GJ Qin, SD Guo, JM Wu, DZ Zhao (2006). Effect of nitrogen fertilizer application on Elymus Nutans seed quality and yield in Qinghai-Tibet plateau. Acta Agrest Sin 14:48-51

Raman G, S Park (2016). The complete chloroplast genome sequence of Ampelopsis: Gene organization, comparative analysis, and phylogenetic relationships to other angiosperms. Front Plant Sci 7; Article 341

Ravi V, JP Khurana, AK Tyagi, P Khurana (2008). An update on chloroplast genomes. Plant Syst Evol 271:101-122

Safonova Y, A Bankevich, PA Pevzner (2015). dipSPAdes: Assembler for highly polymorphic diploid genomes. J Comput Biol 22:528-545

Shah P, MA Gilchrist (2011). Explaining complex codon usage patterns with selection for translational efficiency, mutation bias and genetic drift. Proc Natl Acad Sci USA 108:10231-10236

Toro ND, A Fernandez-Ruiz, L Mignacca, P Kalegari, MC Rowell, S Igelmann, E Saint-Germain, M Benfdil, S Lopes-Paciencia, L Brakier-Gingras (2019). Ribosomal protein RPL22/eL22 regulates the cell cycle by acting as an inhibitor of the CDK4-cyclin D complex. Cell Cycl 18:759-770

Turmel M, JF Pombert, P Charlebois, C Otis, C Lemieux (2007). The green algal ancestry of land plants as revealed by the chloroplast genome. Intl J Plant Sci 168:679-689 
Yu et al. / Intl J Agric Biol, Vol 25, No 6, 2021

Xiong Y, Y Xiong, S Jia, X Ma (2020). The complete chloroplast genome sequencing and comparative analysis of reed canary grass (Phalaris arundinacea) and harding grass (P. aquatica). Plants 9; Article 748

Yamane K, T Kawahara (2018). Size homoplasy and mutational behavior of chloroplast simple sequence repeats (cpSSRs) inferred from intra- and interspecific variations in four chloroplast regions of diploid and polyploid Triticum and Aegilops species. Genet Resour Crop Evol 65:727-743

Zhang D, K Li, J Gao, Y Liu, LZ Gao (2016a). The complete plastid genome sequence of the wild rice Zizania latifolia and comparative chloroplast genomics of the rice tribe Oryzeae, Poaceae. Front Ecol Evol 4; Article 88
Zhang Z, J Zhang, X Zhao, W Xie, Y Wang (2016b). Assessing and broadening genetic diversity of Elymus sibiricus germplasm for the improvement of seed shattering. Molecules 21; Article 869

Zhang Z, W Xie, J Zhang, N Wang, Y Zhao, Y Wang, S Bai (2019). Construction of the first high-density genetic linkage map and identification of seed yield-related QTLs and candidate genes in Elymus sibiricus, an important forage grass in Qinghai-Tibet Plateau. BMC Genomics 20; Article 861

Zuo H, P Wu, D Wu, G Sun (2015). Origin and reticulate evolutionary process of wheatgrass Elymus trachycaulus (Triticeae: Poaceae). PLoS One 10; Article e0125417 